\title{
Buckling of Mechanically Coupled Microbeam Resonators
}

\author{
Bashar Hammad $^{1}$, Ali Nayfeh ${ }^{2,3}$ \\ ${ }^{1}$ German Jordanian University \\ Amman, Jordan \\ bashar.hammad@gju.edu.jo \\ ${ }^{2}$ University of Jordan \\ Amman, Jordan \\ ${ }^{3}$ Virginia Tech \\ Blacksburg, VA, USA
}

\begin{abstract}
We present a methodology to determine analytically buckling loads of mechanically coupled microbeam resonators. To demonstrate how this methodology works, we analyze a structure composed of two beams coupled by a weak coupling beam. We reduce a boundary-value problem composed of five equations and twenty boundary conditions to a set of three linear homogeneous algebraic equations for three constants and the buckling load in order to obtain a deeper insight into the relation between the design parameters and the performance metrics. We study the effect of the residual stress on the static stability of the structure. This methodology can be simply extended to accommodate any boundary conditions and any number of mechanically coupled microbeam resonators (arrays). We found that the critical buckling stress in the coupling beam is very high compared to the residual stresses in the primary beams as long as they are far from the critical values for the primary beams.
\end{abstract}

Keywords: Buckling, Microbeams, Array, Micromechanical Filter, Coupling, Analytical Method.

\section{Introduction}

Mechanically coupled microbeam resonators have attracted special attention in microelectromechanical systems (MEMS). Deflection and natural frequency of these coupled resonators are commonly approximated numerically using Finite-element packages, such as ANSYS, COMSOL, and Coventor. Closed-form analytical solutions for buckling problem of cylindrical shells is derived in [1], and thermal buckling problem for single beams is investigated in [2]. However, to the best of authors' knowledge, there has been few attempt to analytically generate the buckling load of multi-resonator micromechanical structures characterized by distributed-parameter systems.

The fabrication of microresonators in specific and microsystems in general becomes complicated due to buckling induced by residual stresses [3]. Upon finishing the fabrication process, mainly two types of residual stresses are introduced to the final product; thermal and intrinsic stresses [4]. The thermal stress is well understood, whereas the intrinsic stress is more complex and is not well comprehended. The difference in the thermal expansion coefficients of the deposited material and the substrate is the reason behind thermal stress, whereas there are many possible sources for the intrinsic stress. These sources include excess vacancies, dislocations, grain boundaries, phase transformations, precipitation, and compositional changes [4]. Another source of stress is due to gravity, but it can be neglected compared to the mean stress at room temperature [4]. Residual stresses can have significant consequences on the functionality and reliability of micro-structures. The stresses and their gradients are very important for device performance. The resulting stresses can often induce many effects that are undesirable, such as excessive deformation, fracture, delamination, and micro-structural changes in the material that can cause breaking of the structure during fabrication or change the required behavior of the end product $[5,6]$.

To discuss the proposed methodology in this paper and without loss of generality, we present an analytical approach for calculating the buckling of the structure of micromechanical filters made of two clamped-clamped beam resonators connected via a coupling beam. This analytical methodology is easier to handle, more robust, and accurate, and hence allow the designer to obtain a deeper insight into the relationship among performance metrics and the underlying micro-structure dimensions, boundary conditions, and material properties. Moreover, this helps the designer determine the free-buckling operating range for these resonators, especially coupling beams. 


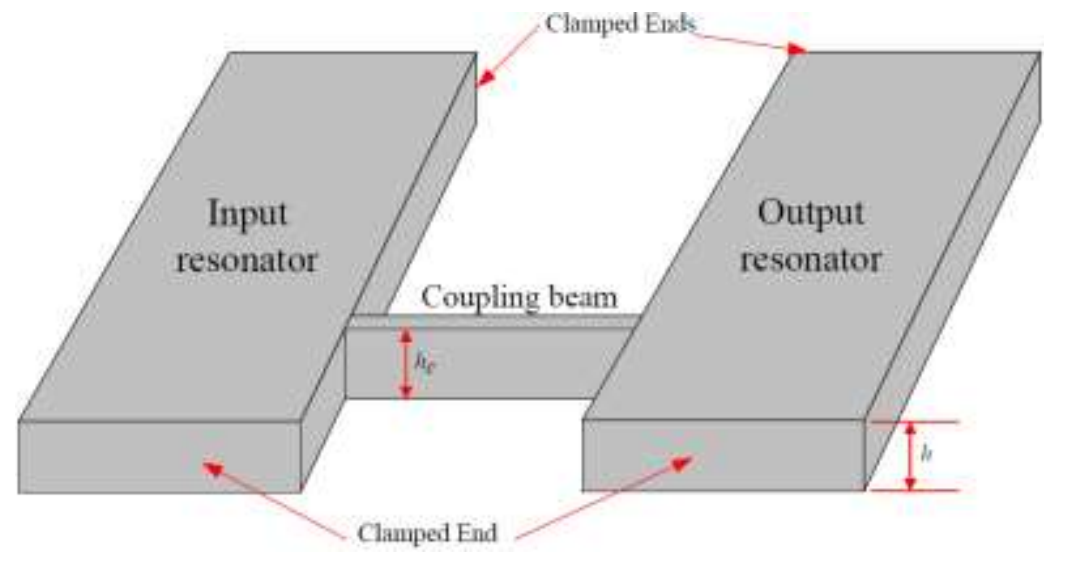

(a)

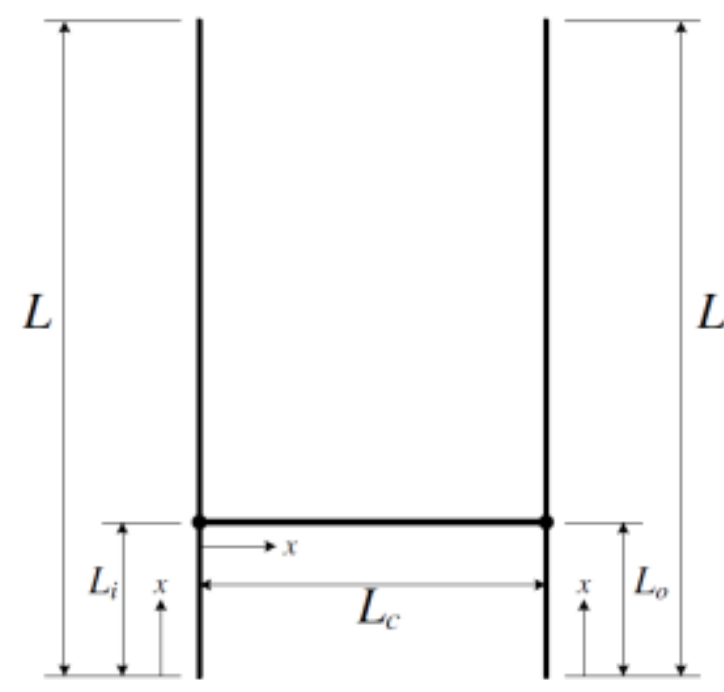

(b)

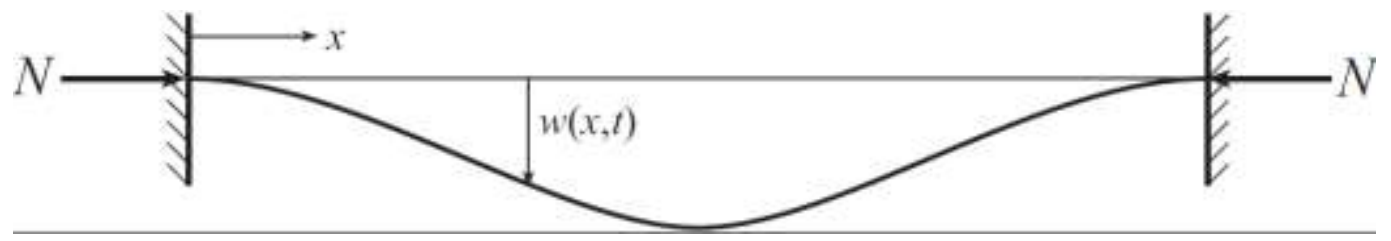

(c)

Fig. 1: (a) A schematic drawing and (b) a schematic model of a structure made of two clamped-clamped microbeam resonators coupled by a weak coupling beam and (c) a schematic diagram for one of the primary resonators.

\section{Problem Formulation}

We compute the buckling load and the associated mode shape of a structure composed of two clamped-clamped microbeam resonators (primary beams) coupled by a weak coupling microbeam, Fig. 1a. Each primary resonator is divided into two parts at the location where the coupling beam is attached to it, Fig. 1b. Consequently, the boundary-value problem (BVP) governing the buckling load is composed of five equations (one equation for each part of the primary beams and one for the coupling beam) and twenty boundary conditions. This problem is transformed into solving a system of twenty linear homogeneous algebraic equations for twenty constants and the buckling load. Using algebraic manipulations, we reduce this problem to that of solving a system of three linear homogeneous algebraic equations for three constants and the buckling load. The determinant of the coefficient matrix of the reduced problem yields the characteristic equation, which is solved for the buckling load. Then, the mode shape is calculated.

\subsection{Governing Equations}

The equations of motion describing the linear, undamped, and unforced deflection of the segments of the primary beams and the coupling beam are [7]

$$
\begin{aligned}
& E I \frac{\partial^{4} \omega_{i, 1}}{\partial x^{4}}+\bar{N}_{\iota} \frac{\partial^{2} \omega_{i, 1}}{\partial x^{2}}+\rho A \frac{\partial^{2} \omega_{i, 1}}{\partial t^{2}}=0 \quad \text { where } \quad 0<x<L_{i} \\
& E I \frac{\partial^{4} \omega_{i, 2}}{\partial x^{4}}+\bar{N}_{\iota} \frac{\partial^{2} \omega_{i, 2}}{\partial x^{2}}+\rho A \frac{\partial^{2} \omega_{i, 2}}{\partial t^{2}}=0 \quad \text { where } \quad L_{i}<x<L
\end{aligned}
$$




$$
\begin{array}{lll}
E I \frac{\partial^{4} \omega_{o, 1}}{\partial x^{4}}+\overline{N_{o}} \frac{\partial^{2} \omega_{o, 1}}{\partial x^{2}}+\rho A \frac{\partial^{2} \omega_{o, 1}}{\partial t^{2}}=0 & \text { where } & 0<x<L_{o} \\
E I \frac{\partial^{4} \omega_{o, 2}}{\partial x^{4}}+\overline{N_{o}} \frac{\partial^{2} \omega_{o, 2}}{\partial x^{2}}+\rho A \frac{\partial^{2} \omega_{o, 2}}{\partial t^{2}}=0 & \text { where } & L_{o}<x<L \\
E I_{c} \frac{\partial^{4} \omega_{c}}{\partial x^{4}}+\overline{N_{c}} \frac{\partial^{2} \omega_{c}}{\partial x^{2}}+\rho A_{c} \frac{\partial^{2} \omega_{c}}{\partial t^{2}}=0 & \text { where } 0<x<L_{c}
\end{array}
$$

where $x$ is the position along each beam's axis, Fig.s $1 \mathrm{~b}$ and $1 \mathrm{c} ; t$ is time; $\omega$ is the downward transverse deflection of the beam and it is a function of $x$ and $t$, Fig. $1 \mathrm{c} ; E$ is Young's modulus; $\rho$ is the material density; $I$ and $I_{c}$ are the moments of inertia of the cross-sections of the primary and coupling beams, respectively; $A$ and $A_{c}$ are the areas of the cross-sections of the primary and coupling beams, respectively; $L_{i}$ and $L_{o}$ are the positions at which the coupling beam is attached to the input and output resonators, respectively; $L$ and $L_{c}$ are the lengths of the primary and coupling beams, respectively; and $\bar{N}$ is the applied compressive axial force. Throughout this paper, the subscripts $i ; o$ and $c$ refer to quantities related to the input, output and coupling beams, respectively. The subscripts 1 and 2 refer to the first and second parts, respectively, of each primary beam. For convenience, we introduce the nondimensional variables

$$
\begin{array}{rcl}
\hat{\omega}=\frac{\omega}{d} & \hat{x}=\frac{x}{L} & \hat{t}=\frac{t}{T} \\
l_{i}=\frac{L_{i}}{L} & l_{o}=\frac{L_{o}}{L} & l_{c}=\frac{L_{c}}{N^{2}} \\
N_{i}=\frac{\overline{N_{l}} L^{2}}{E I} & N_{o}=\frac{\overline{N_{o}} L^{2}}{E I} & N_{c}=\frac{\frac{N_{c}}{E}}{E I_{c}}
\end{array}
$$

where $T=\sqrt{\rho A L^{4} / E I}$ and $d$ is the distance between the beams and electrodes. Substituting equations (6) into equations (1)-(5) and dropping the hats, we obtain

$$
\begin{array}{cccc}
\frac{\partial^{4} \omega_{i, 1}}{\partial x^{4}}+N_{i} \frac{\partial^{2} \omega_{i, 1}}{\partial x^{2}}+\frac{\partial^{2} \omega_{i, 1}}{\partial t^{2}}=0 & \text { where } & 0<x<l_{i} \\
\frac{\partial^{4} \omega_{i, 2}}{\partial x^{4}}+N_{i} \frac{\partial^{2} \omega_{i, 2}}{\partial x^{2}}+\frac{\partial^{2} \omega_{i, 2}}{\partial t^{2}}=0 & \text { where } & l_{i}<x<1 \\
\frac{\partial^{4} \omega_{o, 1}}{\partial x^{4}}+N_{o} \frac{\partial^{2} \omega_{o, 1}}{\partial x^{2}}+\frac{\partial^{2} \omega_{o, 1}}{\partial t^{2}}=0 & \text { where } & 0<x<l_{o} \\
\frac{\partial^{4} \omega_{o, 2}}{\partial x^{4}}+N_{o} \frac{\partial^{2} \omega_{o, 2}}{\partial x^{2}}+\frac{\partial^{2} \omega_{o, 2}}{\partial t^{2}}=0 & \text { where } & l_{o}<x<1 \\
\frac{\partial^{4} \omega_{c}}{\partial x^{4}}+N_{c} \frac{\partial^{2} \omega_{c}}{\partial x^{2}}+\left(\frac{h}{h_{c}}\right)^{2} \frac{\partial^{2} \omega_{c}}{\partial t^{2}}=0 & \text { where } & 0<x<l_{c}
\end{array}
$$

where $h$ and $h_{c}$ are the thicknesses of the primary and coupling beams, respectively, as shown in Fig. 1a.

\subsection{Boundary Conditions}

For the clamped (fixed) ends of the primary beams, the deflections and the slopes vanish; that is, 


$$
\begin{array}{llll}
\omega_{i, 1}(0)=0 & \left.\frac{\partial \omega_{i, 1}}{\partial x}\right|_{x=0}=0 & \omega_{o, 1}(0)=0 & \left.\frac{\partial \omega_{o, 1}}{\partial x}\right|_{x=0}=0 \\
\omega_{i, 2}(1)=0 & \left.\frac{\partial \omega_{i, 2}}{\partial x}\right|_{x=1}=0 & \omega_{o, 2}(1)=0 & \left.\frac{\partial \omega_{o, 2}}{\partial x}\right|_{x=1}=0
\end{array}
$$

At the attachment point in each of the primary beams, the deflection, slope, and moment are continuous. Hence, we have

$$
\begin{aligned}
\omega_{i, 1}\left(l_{i}\right)=\omega_{i, 2}\left(l_{i}\right) & \left.\frac{\partial \omega_{i, 1}}{\partial x}\right|_{x=l_{i}}=\left.\frac{\partial \omega_{i, 2}}{\partial x}\right|_{x=l_{i}} & \left.\frac{\partial^{2} \omega_{i, 1}}{\partial x^{2}}\right|_{x=l_{i}}=\left.\frac{\partial^{2} \omega_{i, 2}}{\partial x^{2}}\right|_{x=l_{i}} \\
\omega_{o, 1}\left(l_{o}\right)=\omega_{o, 2}\left(l_{o}\right) & \left.\frac{\partial \omega_{o, 1}}{\partial x}\right|_{x=l_{o}}=\left.\frac{\partial \omega_{o, 2}}{\partial x}\right|_{x=l_{o}} & \left.\frac{\partial^{2} \omega_{o, 1}}{\partial x^{2}}\right|_{x=l_{o}}=\left.\frac{\partial^{2} \omega_{o, 2}}{\partial x^{2}}\right|_{x=l_{o}}
\end{aligned}
$$

The deflections of the coupling beam are the same as the deflections of the primary beams at the attachment points, and the slopes of the coupling beam at these attachment points vanish. Therefore,

$$
\begin{array}{cc}
\omega_{c}(0)=\omega_{i, 1}\left(l_{i}\right) & \omega_{c}\left(l_{c}\right)=\omega_{o, 1}\left(l_{o}\right) \\
\left.\frac{\partial \omega_{c}}{\partial x}\right|_{x=0}=0 & \left.\frac{\partial \omega_{c}}{\partial x}\right|_{x=l_{c}}=0
\end{array}
$$

The shear forces at the ends of the coupling beam are equal to the changes in the shear forces in the primary beams. These conditions yield

$$
\begin{gathered}
\left.\frac{\partial^{3} \omega_{i, 1}}{\partial x^{3}}\right|_{x=l_{i}}-\left.\frac{\partial^{3} \omega_{i, 2}}{\partial x^{3}}\right|_{x=l_{i}}=\left.\frac{I_{c}}{I} \frac{\partial^{3} \omega_{c}}{\partial x^{3}}\right|_{x=0} \\
\left.\frac{\partial^{3} \omega_{o, 1}}{\partial x^{3}}\right|_{x=l_{o}}-\left.\frac{\partial^{3} \omega_{o, 2}}{\partial x^{3}}\right|_{x=l_{o}}=-\left.\frac{I_{c}}{I} \frac{\partial^{3} \omega_{c}}{\partial x^{3}}\right|_{x=l_{c}}
\end{gathered}
$$

\section{Buckling Problem}

The buckling problem can be obtained from equations (7)-(11) by dropping the time derivatives and replacing $\omega_{i}, \omega_{o}$, and $\omega_{c}$ with the functions $\psi_{i}, \psi_{o}$, and $\psi_{c}$, which are the static configurations associated with the forces $N_{i}, N_{o}$, and $N_{c}$, respectively [8]. The resulting equations are

$$
\begin{aligned}
& \psi_{i, 1}^{i v}(x)+N_{i} \psi_{i, 1}^{\prime \prime}(x)=0 \quad \text { where } 0<x<l_{i} \\
& \psi_{i, 2}^{i v}(x)+N_{i} \psi_{i, 2}^{\prime \prime}(x)=0 \quad \text { where } l_{i}<x<1 \\
& \psi_{o, 1}^{i v}(x)+N_{o} \psi_{o, 1}^{\prime \prime}(x)=0 \quad \text { where } 0<x<l_{o} \\
& \psi_{o, 2}^{i v}(x)+N_{o} \psi_{o, 2}^{\prime \prime}(x)=0 \quad \text { where } l_{o}<x<1 \\
& \psi_{c}^{i v}(x)+N_{c} \psi_{c}^{\prime \prime}(x)=0 \quad \text { where } 0<x<l_{c}
\end{aligned}
$$

subject to the following boundary conditions (discussed in details in Section 2.2): 
- $\quad$ for the clamped edges

$$
\begin{array}{llll}
\psi_{i, 1}(0)=0 & \psi^{\prime}{ }_{i, 1}(0)=0 & \psi_{o, 1}(0)=0 & \psi_{o, 1}^{\prime}(0)=0 \\
\psi_{i, 2}(1)=0 & \psi^{\prime}{ }_{i, 2}(1)=0 & \psi_{o, 2}(1)=0 & \psi_{o, 2}^{\prime}(1)=0
\end{array}
$$

- $\quad$ at the attachment points in the primary beams

$$
\begin{aligned}
\psi_{i, 1}\left(l_{i}\right)=\psi_{i, 2}\left(l_{i}\right) & \psi^{\prime}{ }_{i, 1}\left(l_{i}\right)=\psi^{\prime}{ }_{i, 2}\left(l_{i}\right) & \psi^{\prime \prime}{ }_{i, 1}\left(l_{i}\right)=\psi^{\prime \prime}{ }_{i, 2}\left(l_{i}\right) \\
\psi_{o, 1}\left(l_{o}\right)=\psi_{o, 2}\left(l_{o}\right) & \psi^{\prime}{ }_{o, 1}\left(l_{o}\right)=\psi^{\prime}{ }_{o, 2}\left(l_{o}\right) & \psi^{\prime \prime}{ }_{o, 1}\left(l_{o}\right)=\psi_{o, 2}^{\prime \prime}\left(l_{o}\right)
\end{aligned}
$$

- at the attachment points in the coupling beam

$$
\begin{array}{cc}
\psi_{c}(0)=\psi_{i, 1}\left(l_{i}\right) & \psi_{c}\left(l_{c}\right)=\psi_{o, 1}\left(l_{o}\right) \\
\psi^{\prime}{ }_{c}(0)=0 & \psi^{\prime}{ }_{c}\left(l_{c}\right)=0
\end{array}
$$

- $\quad$ and the shear force at the attachment points

$$
\begin{aligned}
\psi^{\prime \prime \prime}{ }_{i, 1}\left(l_{i}\right)-\psi^{\prime \prime \prime}{ }_{i, 2}\left(l_{i}\right) & =\frac{I_{c}}{I} \psi^{\prime \prime \prime}{ }_{c}(0) \\
\psi^{\prime \prime \prime}{ }_{o, 1}\left(l_{o}\right)-\psi^{\prime \prime \prime}{ }_{o, 2}\left(l_{o}\right) & =-\frac{I_{c}}{I} \psi^{\prime \prime \prime}{ }_{c}\left(l_{c}\right)
\end{aligned}
$$

The general solutions of equations (20)-(24) are given by

$$
\begin{array}{lrr}
\psi_{i, 1}(x)=c_{1}+c_{2} x+c_{1 a} \cos \left(\sqrt{N_{i}} x\right)+c_{2 a} \sin \left(\sqrt{N_{i}} x\right) & \text { where } & 0<x<l_{i} \\
\psi_{i, 2}(x)=c_{3}+c_{4}(1-x)+c_{3 a} \cos \left(\sqrt{N_{i}}(1-x)\right)+c_{4 a} \sin \left(\sqrt{N_{i}}(1-x)\right) & \text { where } & l_{i}<x<1 \\
\psi_{o, 1}(x)=c_{5}+c_{6} x+c_{5 a} \cos \left(\sqrt{N_{o}} x\right)+c_{6 a} \sin \left(\sqrt{N_{o}} x\right) & \text { where } & 0<x<l_{o} \\
\psi_{o, 2}(x)=c_{7}+c_{8}(1-x)+c_{7 a} \cos \left(\sqrt{N_{o}}(1-x)\right)+c_{8 a} \sin \left(\sqrt{N_{o}}(1-x)\right) & \text { where } & l_{o}<x<1 \\
\psi_{c}(x)=c_{9}+c_{10} x+c_{11} \cos \left(\sqrt{N_{c}} x\right)+c_{12} \sin \left(\sqrt{N_{c}} x\right) & \text { where } & 0<x<l_{c}
\end{array}
$$

Substituting equations (33)-(37) into equations (25)-(32) yields a system of twenty linear homogeneous algebraic equations in the twenty unknown coefficients $c_{1}, c_{2}, \ldots, c_{12}$ and $c_{1 a}, c_{2 a}, \ldots, c_{8 a}$, which can be written in matrix form as

$$
M_{B} \boldsymbol{c}=\mathbf{0}
$$

where $M_{B}$ is a $20 \times 20$ matrix called the coefficient matrix, $c$ is a $20 \times 1$ vector whose elements are the above unknown coefficients, and $\boldsymbol{0}$ is a $20 \times 1$ zero vector. The elements of $M_{B}$ are functions of $N_{i}, N_{o}$, and $N_{c}$, and the dimensions of the structure. Substituting equations (33)-(37) into equation (25)-(26) and solving for the $c_{j a}$ in terms of the $c_{j}$, we obtain

$$
\psi_{i, 1}(x)=c_{1}\left\{1-\cos \left(\sqrt{N_{i}} x\right)\right\}+c_{2}\left\{x-\left(1 / \sqrt{N_{i}}\right) \sin \left(\sqrt{N_{i}} x\right)\right\} \quad \text { where } 0<x<l_{i}
$$




$$
\begin{array}{ll}
\psi_{i, 2}(x)=c_{3}\left\{1-\cos \left(\sqrt{N_{i}}(1-x)\right)\right\}+c_{4}\left\{(1-x)-\left(1 / \sqrt{N_{i}}\right) \sin \left(\sqrt{N_{i}}(1-x)\right)\right\} & \text { where } l_{i}<x<1 \\
\psi_{o, 1}(x)=c_{5}\left\{1-\cos \left(\sqrt{N_{o}} x\right)\right\}+c_{6}\left\{x-\left(1 / \sqrt{N_{o}}\right) \sin \left(\sqrt{N_{o}} x\right)\right\} & \text { where } 0<x<l_{o} \\
\psi_{o, 2}(x)=c_{7}\left\{1-\cos \left(\sqrt{N_{o}}(1-x)\right)\right\}+c_{8}\left\{(1-x)-\left(1 / \sqrt{N_{o}}\right) \sin \left(\sqrt{N_{o}}(1-x)\right)\right\} & \text { where } l_{o}<x<1 \\
\psi_{c}(x)=c_{9}+c_{10} x+c_{11} \cos \left(\sqrt{N_{c}} x\right)+c_{12} \sin \left(\sqrt{N_{c}} x\right) & \text { where } 0<x<l_{c}
\end{array}
$$

We substitute equations (39)-(43) into equations (27), (28), (30), and (31) and solve the resulting nine equations for the $c_{j}$ in terms of $c_{1}, c_{5}$, and $c_{9}$, which represent the input, output, and coupling beams, respectively. Then, using the remaining three boundary conditions, equations (29) and (32), we obtain the reduced problem

where

$$
M_{B, r} \boldsymbol{c}_{\boldsymbol{r}}=\mathbf{0}
$$

$$
M_{B, r}=\left[\begin{array}{lll}
f_{11} & f_{12} & f_{13} \\
f_{21} & f_{22} & f_{23} \\
f_{31} & f_{32} & f_{33}
\end{array}\right] \quad \quad \boldsymbol{c}_{\boldsymbol{r}}=\left(\begin{array}{c}
c_{1} \\
c_{5} \\
c_{9}
\end{array}\right)
$$

The zero vector $\mathbf{0}$ in this case is a $3 \times 1$ vector and the elements $f_{m n}$ are functions of $N_{i}, N_{o}$, and $N_{c}$. Setting the determinant of $M_{B, r}$ equal to zero yields the characteristic equation

$$
\operatorname{Det}\left[M_{B, r}\right]=0
$$

We substitute two of the applied compressive forces $N_{i}, N_{o}$, and $N_{c}$ into the above characteristic equation, then solve it for the third force of the structure. In Section 5 (Results and Discussion), we specify $N_{i}$ and $N_{o}$ for reasons explained in that section and solve for $N_{c}$. For each set of $N_{i}, N_{o}$, and $N_{c}$ satisfying equation (46), there is a corresponding mode shape. To calculate this mode shape, we substitute the numerical values for $N_{i}, N_{o}$, and $N_{c}$, into equations (39)-(43). Then, we use the boundary conditions to find numerical relations among the unknowns and end up with

$$
a_{1} c_{1}+a_{2} c_{5}=0
$$

where $a_{1}$ and $a_{2}$ are numerical constants relating the unknowns $c_{1}$ and $c_{5}$ obtained after substituting numerical values for $N_{i}, N_{o}$, and $N_{c}$ and boundary conditions. By setting $c_{1}$ equal to one, we find numerical values for all of the $c$ 's in equations (39)-(43), and hence the mode shape corresponding to that set of $N_{i}, N_{o}$, and $N_{c}$.

\section{Normalization of Mode Shapes}

Because the algebraic system of equations, equation (38), is homogeneous, it follows that if the vector $\mathrm{c}$ is a solution of the equation, then $\alpha c$ is also a solution, where $\alpha$ is an arbitrary constant. This implies that the mode shape is unique to within a constant. To normalize the mode shape, i.e., to render it unique, a very convenient normalization scheme of the mode shape consists of setting [9]

$$
\int_{x_{1}}^{x_{2}}(\alpha \psi(x))^{2} d x=1
$$

where $\psi(x)$ is the mode shape. Hence, the constant $\alpha$ is given by 


$$
\alpha=\frac{1}{\sqrt{\int_{x_{1}}^{x_{2}} \psi(x)^{2} d x}}
$$

Considering the topology of the structure investigated in this work and the analysis of the reduced-order model performed in [10], the $\alpha$ constant associated with $\psi$ and $N$ is given by

$$
\alpha=\left(\int_{0}^{l_{i}} \psi_{i, 1}^{2}(x) d x+\int_{l_{i}}^{1} \psi_{i, 2}^{2}(x) d x+\int_{0}^{l_{o}} \psi_{o, 1}^{2}(x) d x+\int_{l_{o}}^{1} \psi_{o, 2}^{2}(x) d x+T_{c}^{2} \int_{0}^{l_{c}} \psi_{c}^{2}(x) d x\right)^{-\frac{1}{2}}
$$

The parameter $T_{c}^{2}$ is the ratio of the cross-section area of the coupling beam to that of the primary beam, $A_{c} / A$.

\section{Results and Discussion}

We utilize the developed methodology to study the structure of filter fabricated by Bannon et al [11], but without considering the frequency modification factor or any adjustments due to fabrication processes. In this section, we compute the buckling load and plot the corresponding mode shape. The design parameters, dimensions, and material properties of the structure used in this analysis are obtained from [11] and listed in Table 1.

In this work, we model the residual stress as an axial load applied to the beam [12-14]. Typically, the residual stress is of the tensile type in microbeam resonators and of the compressive type if it is induced by temperature variations in these microbeams [15]. This implies that we have to ensure that all beams operate away from the critical buckling stress. Using the methodology discussed in Section 3, we assume that the axial load in the input and output beams are the same (i.e., $N_{i}=$ $N_{o}$ ) and we vary their values from the state of free residual stress to the state where a clamped-clamped beam buckles. Then, we solve the characteristic equation, equation (46), to obtain the buckling load $N_{c}$ in the coupling beam. Because the coupling beam is weak, its effect on the buckling load of the primary beams is negligible. Therefore, the critical nondimensional buckling load in the input and output beams is given by the following formula for a clamped-clamped beam $[8,16]$ :

$$
N_{c r}=4 n \pi^{2}
$$

where $n$ is an integer denoting the buckling mode. Because we are interested in buckling when it occurs for the first time (i.e., $n=1$ ), we found that $N_{c r}=39.48$ (in terms of dimensional quantities $\bar{N}_{c r} \cong 0.0163 \mathrm{~N}$ ).

Using the specifications listed in Table 1 and solving equation (46), as the axial force in the input and output beams is increased (and keeping $N_{i}=N_{o}$ ), the buckling load in the coupling beam $N_{c}$ does not change significantly and continue to have a value of $N_{c}=323.55$ as long as $N_{i}$ and $N_{o}$ are far from their critical buckling load $N_{c r}=39.48$, Fig. 2. In this case (far below $N_{c r}$ ) the mode shapes calculated based on equations (39)-(43) with normalization as explained in Section 4 become as depicted in Fig.s 3 a. But as $N_{i}$ and $N_{o}$ become closer to the critical value $N_{c r}, N_{c}$ drops rapidly until the input and output beams buckle at their critical load $N_{c r}=39.48$, and the mode shape in the case becomes as shown in Fig. 3b. Consequently, for any $N_{i}$ and $N_{o}$ values lower than $N_{c r}$, the coupling beam buckles when the axial force exceeds $N_{c}$ as shown in Fig. 2. Fig.s $4 \mathrm{a}$ and $\mathrm{b}$ show the same result obtained in Fig. 2 but in terms of dimensional quantities where we use equation (6) to obtain the axial force in terms of Newtons, Fig. 4a, and we then divide this axial force by the cross-section areas of the primary and coupling beams to express the result in terms of axial stresses, Fig. 4b. From these figures, we observe that the buckling stress in the coupling beam is very large when the axial stresses are much lower than the critical value of buckling stress in the primary beams.

It is important to note that the model for the buckling problem presumes implicitly that the motions in the in-plane direction are neglected. However, for the structure and dimensions described in this paper, and in case the motion of the coupling beam in the in-plane direction is taken into account, the coupling beam buckles in the in-plane direction. This is due to the fact that the flexural stiffness $E I$ of the coupling beam in the in-plane direction is smaller than that in the transverse direction. Because the coupling beam in the in-plane direction can be approximated by a clamped-clamped beam (primary beams are very stiff in the longitudinal direction), the critical buckling load of the coupling beam in the in-plane direction is $\bar{N}_{c, c r} \cong 0.0010 \mathrm{~N}$, which corresponds to the stress $\sigma_{c, c r} \cong 670 \mathrm{MPa}$. However, we have assumed ideal clamped-clamped boundary conditions in this paper. In reality, these ends have finite rigidity and some structures have step-up type [17] or 
special design [11] supports. Consequently, the actual buckling loads are slightly different than those of a microbeam with ideal clamped-clamped ends.

Table 1: Structure specifications [11].

\begin{tabular}{|l|c|}
\hline \multicolumn{1}{|c|}{ Parameter } & Design Value \\
\hline Primary Resonator Length, $L(\mu \mathrm{m})$ & 40.8 \\
\hline Primary Resonator Width, $b(\mu \mathrm{m})$ & 8.0 \\
\hline Coupling Location, $L_{i} \& L_{o}(\mu \mathrm{m})$ & 4.08 \\
\hline Coupling Beam Length, $L_{c}(\mu \mathrm{m})$ & 20.35 \\
\hline Coupling Beam Width, $b_{c}(\mu \mathrm{m})$ & 0.75 \\
\hline Structural Thickness, $h(\mu \mathrm{m})$ & 1.9 \\
\hline Young's Modulus, $E(\mathrm{GPa})$ & 150 \\
\hline Polysilicon Density, $\rho\left(\mathrm{kg} / \mathrm{m}^{3}\right)$ & 2300 \\
\hline
\end{tabular}

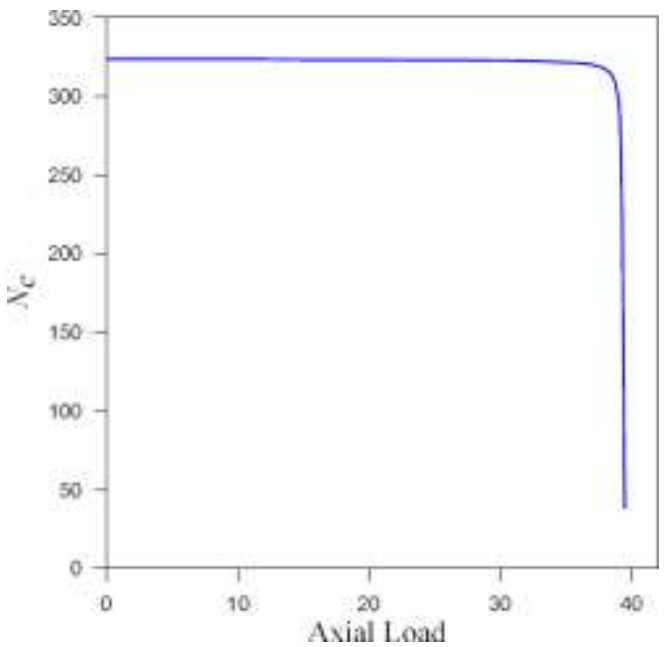

Fig. 2: Variation of the nondimensional buckling load in the coupling beam with the axial load in the primary beams.

\section{Conclusions}

We solved the buckling problem of the structure of micromechanical filters and obtained analytical expression for their buckling load and mode shape. The model described in this work treats the structure as a distributed-parameter system. For a micromechanical structure made of two clamped-clamped beam resonators connected via a coupling beam, we solved a boundary-value problem (BVP) composed of five equations and twenty boundary conditions for its buckling load and mode shapes. We reduced the problem to a set of three linear homogeneous algebraic equations for three constants and the buckling load. Setting the determinant of the reduced coefficient matrix equal to zero, we obtained the characteristic equation, which we solved for buckling load. Then, we computed the constants and hence the mode shapes. The closed-form expressions obtained in this work are easier to handle, more robust, and accurate. We investigated the effect of the residual stresses in the primary beams on the buckling load in the coupling beam. We found that the buckling stress in the coupling beam is very high compared to the residual stresses in the primary beams as long as they are far from the critical buckling values for the primary beams. Finally, we emphasize that although the model presented in this paper for the buckling problems is restricted to micromechanical structure made of two clamped-clamped beam resonators connected via a weak coupling beam, they can be easily modified and adjusted to model any mechanically coupled microbeam arrays, such as, but not limited to, free-free microbeam array, higher-order structures, and arrays with bridges between nonadjacent resonators [18]. 

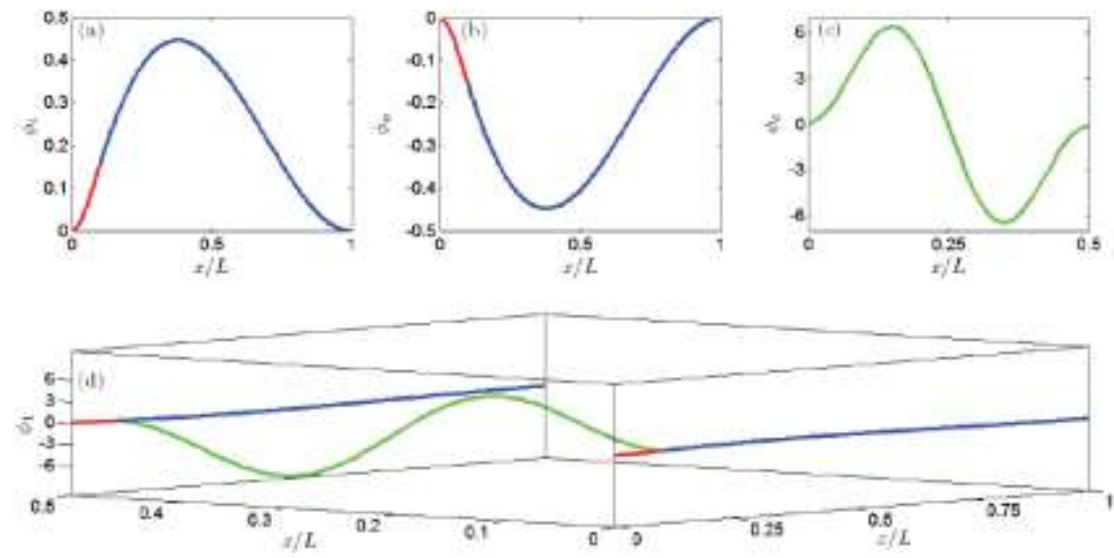

(a)
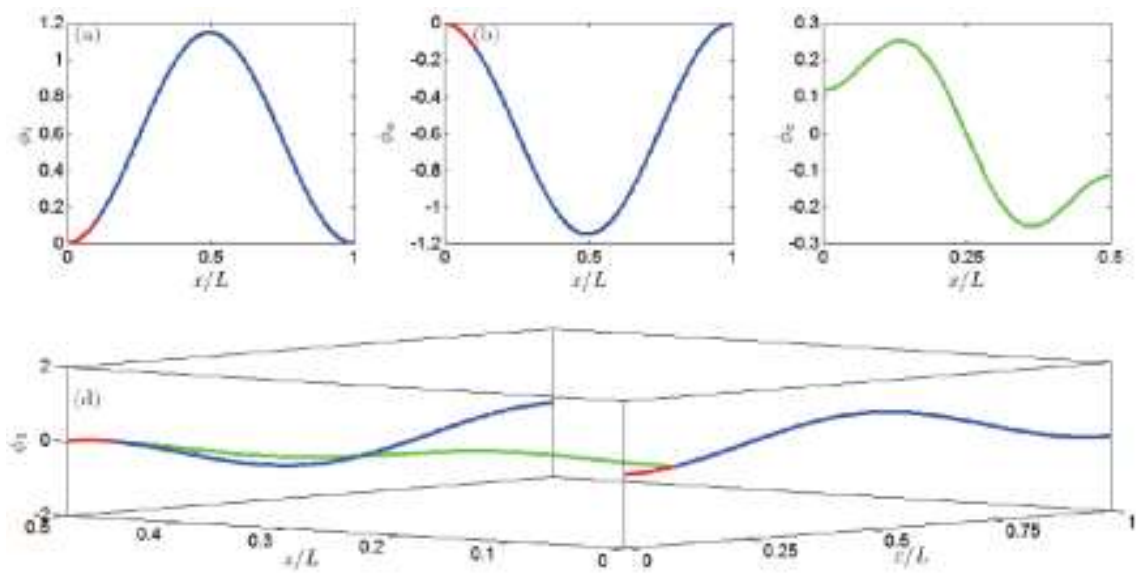

(b)

Fig. 3: Buckling mode shape for (a) $N_{i}=N_{o}=10$ and $N_{c}=323.55$ and (b) $N_{i}=N_{o}=39$ and $N_{c}=298.01$ : (a) input, (b) output, (c) coupling beams, and (d) combination of Fig.s (a), (b), and (c).
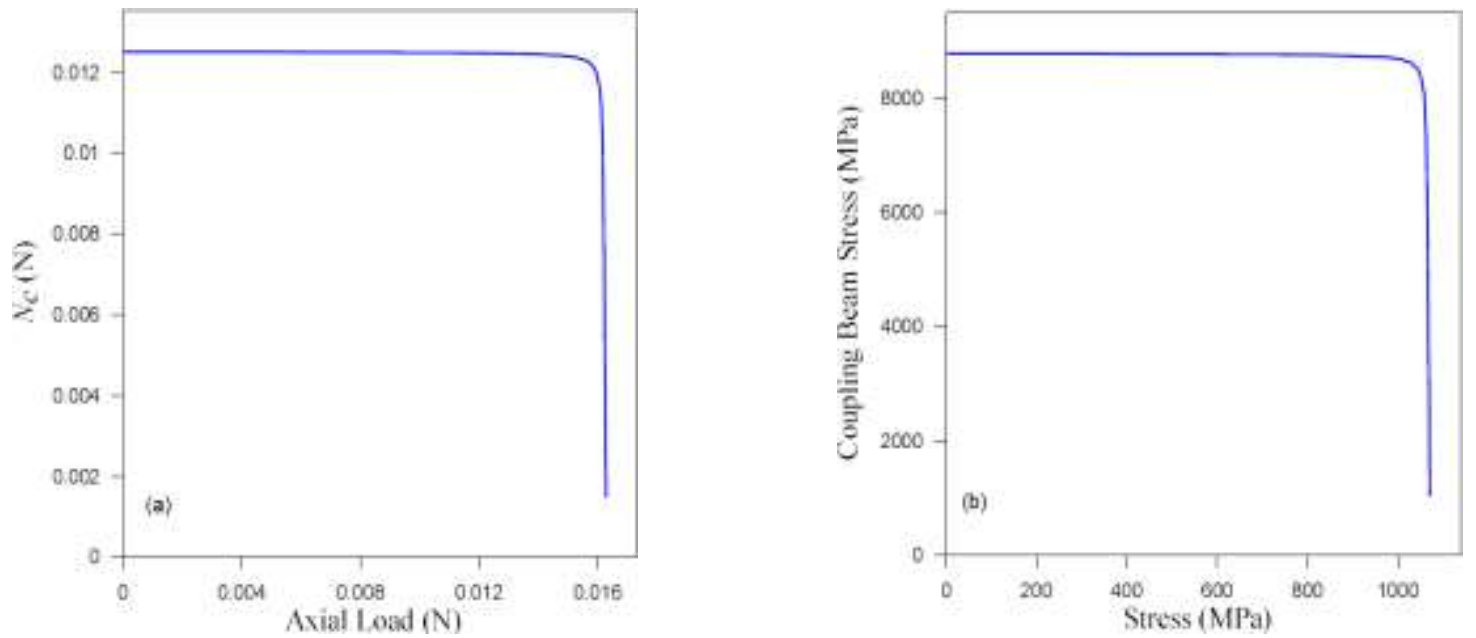

Fig. 4: (a) Variations of the (a) buckling load and (b) the buckling stress in the coupling beam with the axial load in the primary beams. 


\section{References}

[1] M. Lagos and R. Das, "Analytical solution for the axisymmetric buckling of cylindrical shells," International Journal of Mechanics and Materials in Design, vol. 11, no. 2, pp.139-148, 2015.

[2] Y. Kiani and M. R. Eslami, "Thermal buckling analysis of functionally graded material beams," International Journal of Mechanics and Materials in Design, vol. 6, no. 3, pp. 229-238, 2010.

[3] D. Peroulis, S. P. Pacheco, K. Sarabandi and L. P. B. Katehi, "Alleviating the adverse effects of residual stress in RF MEMS switches," in Proc. IEEE 31st European Microwave Conference, pp. 1-4, 2001.

[4] S. Chen, T. V. Baughn, Z. J. Yao and C. L. Goldsmith, "A new in Situ Residual stress measurement method for a MEMS thin fixed-fixed beam structure," JMEMS, vol. 11, no. 4, pp. 309-316, 2002.

[5] L. A. Starman, E. M. Ochoa, J. A. Lott, M. S. Amer, W. D. Cowan, and J. D. Busbee, "Residual stress characterization in MEMS microbridges using micro-Raman spectroscopy," in Proc. NSTI-Nanotech, Int. conference on modeling and simulating of microsystems, vol. 1, pp. 314-317, 2002.

[6] N. Sabate, D. Vogel, A. Gollhardt, J. Keller, C. Cane, I. Gracia, J. R. Morante and B. Michel, "Residual stress measurement on a MEMS structure with high-spatial resolution," JMEMS, vol. 16, no. 2, pp. 365-372, 2007.

[7] B. K. Hammad, "Natural Frequencies and Mode Shapes of Mechanically Coupled Microbeam Resonators with an Application to Micromechanical Filters," Shock and Vibration, 2014.

[8] A. H. Nayfeh and P. F. Pai, Linear and Nonlinear Structural Mechanics. Wiley, New York, 2004.

[9] L. Meirovitch, Fundamentals of Vibrations. McGraw-Hill, 2001.

[10] B. K. Hammad, E. M. Abdel-Rahman and A. H. Nayfeh, "Modeling and analysis of electrostatic MEMS filters," Nonlinear Dynamics, vol. 60, no. 3, pp. 385-401, 2010.

[11] F. D. Bannon, J. R. Clark and C. T.-C. Nguyen, "High-Q HF microelectromechanical filters, " J. Solid-State Circuits, vol. 35, no. 4, pp. 512-526, 2000.

[12] E. M. Abdel-Rahman, M. I. Younis and A. H. Nayfeh, "Characterization of the mechanical behavior of an electrically actuated microbeam," Journal of Micromechanics and Microengineering, vol. 12, no. 6, pp. 759-766, 2002.

[13] Y. Zhang and Y.-P., "Numerical and analytical study on the pull-in instability of micro-structure under electrostatic loading," Sensors and Actuators A: Physical, vol. 127, no. 2, pp. 366-380, 2006.

[14] M. I. Younis, E. M. Abdel-Rahman and A. H. Nayfeh, "A Reduced-order model for electrically actuated microbeambased MEMS," JMEMS, vol. 12, no. 5, pp. 672-680, 2003.

[15] M. I. Younis and A. H. Nayfeh, "A Study of the nonlinear response of a resonant microbeam to an electric actuation," Nonlinear Dynamics, vol. 31, no. 1, pp. 91-117, 2003.

[16] J. M. Gere, Mechanics of Materials, Brooks/Cole, 2001.

[17] Q. Meng, M. Mehregany and R. Mullen, "Theoretical modeling of microfabricated beams with elastically restrained supports," JMEMS, vol. 2, no. 3, pp. 128-137, 1993.

[18] S.-S. Li, M. U. Demirci, Y.-W. Lin, Z. Ren and C. T.-C. Nguyen, "Bridged micromechanical filters," in Proc. IEEE Int. Ultrasonics, Ferroelectrics, and Frequency Control Joint 50 Anniversary Conference, Montreal, Canada, Aug. 24-27, pp. 280-286, 2004. 